\title{
Competencia socioemocional y toma de decisiones en la deserción
} escolar de estudiantes universitarios

Socio-emotional skills and decision-making in the school dropout of university students

Recepción del artículo: 16-12-2020 ～Aceptación del artículo: 10-02-2021

\section{Resumen}

Mayra, Delgado Villalobos ${ }^{1}$ mayra.delgado@uaem.mx

https://orcid.org/0000-0003-2109-8839

Gerardo, Maldonado Paz'

gerardo.maldonado@uaem.mx

https://orcid.org/0000-0001-7612-2205

Jean, Philippe Jazé1

jphjaze07@gmail.com

https://orcid.org/0000-0001-5446-5872

${ }^{1}$ Centro de Investigación en Ciencias Cognitivas.

Universidad Autónoma del Estado de Morelos

Para referenciar este artículo:

Delgado-Villalobos, M., Maldonado-Paz, G. y Jazé, J. P. (2021). Competencia socioemocional y toma de decisiones en la deserción de estudiantes universitarios. Revista ConCiencia EPG, 6(2), 13-29. https://doi.org/10.32654/CONCIENCIAEP

G.6-2.2

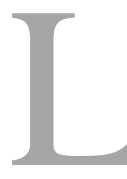

a educación universitaria enfrenta el reto de promover el desarrollo integral del estudiante y su aprendizaje a lo largo de la vida. La competencia socioemocional tiene un impacto positivo en la experiencia de aprendizaje, en el entorno e interacción de los estudiantes y en el éxito de sus estudios. El presente trabajo tuvo como objetivo proponer un modelo estructural que perfile la relación entre competencia socioemocional y toma de decisiones sobre el rendimiento académico de estudiantes universitarios.

El estudio fue exploratorio y transversal. La muestra fue no aleatoria e intencional con 227 estudiantes (156 mujeres, 71 hombres, promedio de edad 21.52 años).

Se utilizó modelaje con ecuaciones estructurales y se identificó que, en la competencia socioemocional, a mayor resiliencia menor regulación emocional. En la toma de decisiones hubo una correlación positiva entre el componente emocional y el componente cognitivo. Se concluye que, desde la cognición social, las interacciones sociales promovidas por las competencias socioemocionales integran al estudiante en su entorno educativo; el componente cognitivo de la toma de decisiones eleva en el estudiante su capacidad de análisis y síntesis de la información para responder de manera positiva a las demandas de contextos complejos y tener un mayor rendimiento académico. 
Palabras Clave: competencia socioemocional, toma de decisiones, rendimiento académico, deserción escolar, educación superior.

\section{Abstract}

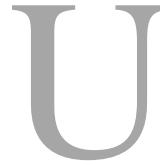

niversity education faces the challenge of promoting the integral development of the student and their learning throughout life. Socio-emotional competence has a positive impact over the learning experience, the environment and interaction of students, and the success of their studies. The objective of this research was to propose a structural model that outlines the relationship between socioemotional competence and decisionmaking on the academic performance of university students.

The study was exploratory and crosssectional. The sample was non-random and intentional with 227 students (156 women, 71 men, mean age 21.52 years).

Modeling with structural equations was used and it was identified that, in socioemotional competence, the less resilience the greater the emotional regulation. In decision making, there was a positive correlation between the emotional component and the cognitive component. It is concluded that, from social cognition, social interactions promoted by socioemotional competences integrate the student in their educational environment; The cognitive component of decisionmaking increases in the student the ability to analyze and synthesize information to respond positively to the demands of complex contexts and have a higher academic performance.

Key Words: socio-emotional competence; decision making; academic performance; school dropouts; undergraduate level.

\section{Introducción}

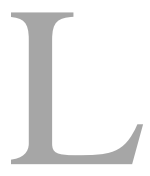

a publicación de la Declaración Mundial sobre la Educación Superior en el siglo XXI, Visión y Acción (1998) exigió a los países generar reformas en profundidad, así como una política de ampliación del acceso a la educación superior. En México, durante los últimos cinco años, la tendencia ha sido un crecimiento y diversificación geográfica de la matrícula en la educación superior; anualmente ha incrementado $3.81 \%$ en promedio. Esto significó, que para el ciclo escolar 2018-2019, la cobertura fuera de $4,344,133$ matrículas exactamente. No obstante, también se ha mantenido el aumento en la deserción escolar, con un promedio del $7.62 \%$ en cada periodo escolar. Sobre esto último, Himmel (2002) define la deserción escolar, como el abandono prematuro que el estudiante hace a un programa de estudios antes de alcanzar el título o grado, considerando un tiempo suficientemente largo para descartar la posibilidad de que se reincorpore. Este fenómeno se puede presentar de dos formas: cuando el estudiante suspende sus estudios y se 
matricula en otro programa de la misma $u$ otra institución, o permanentemente, cuando el estudiante abandona de manera definitiva la educación superior.

Por otra parte, Tinto (1975), a diferencia de Himmel, considera que la deserción es un proceso longitudinal, y centra este proceso en la interacción, integración y adaptación del estudiante a los sistemas sociales de la institución, de tal forma que sus experiencias le permitan modificar continuamente sus metas $y$ compromisos institucionales para permanecer o abandonar la universidad. Así, la deserción escolar está asociada a factores sociales, económicos, familiares, educativos e individuales (Ayala \& Atencio, 2018).

La educación universitaria enfrenta retos y desafíos para impulsar un modelo de enseñanza centrado en el estudiante, por ello, deberá generar estrategias que contribuyan tanto a su formación profesional como a disminuir el abandono escolar. Una de estas estrategias son las competencias, es decir, el potencial de pensamiento y acción que el estudiante debe dominar y mantener durante el proceso de aprendizaje a lo largo de la vida. De acuerdo con Bisquerra (2003), este potencial se categoriza en 1) un saber, representado por el dominio de conocimientos teóricos y científicos; y 2) un saber ser, relacionado al desarrollo como persona, e implica aspectos cognitivos, metodológicos y socioemocionales.

Bisquerra y Pérez (2007) plantean que la competencia socioemocional es la capacidad que se desarrolla a lo largo de la vida para movilizar adecuadamente un conjunto de conocimientos, capacidades, habilidades $\mathrm{y}$ actitudes necesarias para realizar actividades diversas con un cierto nivel de calidad y eficacia, la cual, siempre se puede mejorar. Asimismo, con base en resultados de investigación en los campos de la psicología, la educación, la economía y las neurociencias (Chernyshenko, Kankaraš y Drasgow, 2018; Kautz et al., 2014; De Fruyt, Wille \& John, 2015; Almlund et al., 2011), se considera que las habilidades socioemocionales son maleables y están relacionadas con resultados de éxito de las personas.

De igual forma, existe evidencia de que el aprendizaje de habilidades socioemocionales, tales como el autoconocimiento, la autorregulación, la conciencia social, la colaboración y la toma responsable de decisiones, contribuyen a la prevención de situaciones de riesgo en los jóvenes, al logro de trayectorias laborales exitosas, a generar un clima escolar positivo y a lograr un mejor desempeño académico (Secretaría de Educación Pública, 2020). El desempeño académico se puede definir como una medición de las capacidades que presenta un estudiante sobre lo que ha aprendido, como efecto de un proceso de formación y a la participación de una situación educativa; es un indicador del nivel de aprendizaje logrado por el estudiante en función de objetivos establecidos (Pizarro, 1985; Isaza \& Henao, 2012). También se considera multicausal, porque intervienen componentes tanto internos como externos al individuo, los cuales pueden ser de orden social, cognitivo y emocional, clasificándose en tres categorías: determinantes personales, 
determinantes sociales y determinantes institucionales (Garbanzo, 2007)

Dentro de los determinantes personales, Gaxiola, González y Gaxiola (2013) identificaron que algunas competencias socioemocionales en adolescentes mexicanos, como la autorregulación emocional y conductual, con un coeficiente de correlación de 0.34 sobre la disposición a la resiliencia, predijo positivamente las metas educativas (coeficiente de 0.61) y éstas últimas predijeron significativa y positivamente el rendimiento académico con un coeficiente de 0.41. Asimismo, Velasco, Suárez, Córdova, Luna y Mireles (2015) evidenciaron que un $63 \%$ de los estudiantes de enfermería que presentaban un nivel de resiliencia alto, tenían altos niveles de rendimiento académico: no repetían asignaturas y no tenían exámenes extraordinarios. Otra competencia socioemocional importante es la conducta prosocial, definida como un conjunto de comportamientos destinados a beneficiar a otros, como son la ayuda intra grupal, el voluntariado y la cooperación (Eisenberg \& Fabes, 1998). Estudios empíricos demuestran que, en edades tempranas, esta competencia constituye un predictor significativo del rendimiento académico en edades posteriores (Adeyemi, 2008; Brody et al, 2006; Dana, Scott \& Sunita, 2006; Inglés, et. al, 2009).

En cuanto a la relación entre la emoción y la cognición, las emociones están integradas por procesos psicológicos y por supuesto, por sus correspondientes correlatos neurológicos, lo cual ha derivado en trabajos dirigidos a indagar sobre cómo operan las emociones en procesos tales como la memoria, el lenguaje, la atención o la toma de decisiones; esto por considerar que las emociones son productos surgidos de la interpretación cognitiva (Maldonado, 2014).

Desde la perspectiva cognitiva, la emoción y la toma de decisiones se han abordado en función de las capacidades de control del comportamiento, es decir, mediante el constructo de las funciones ejecutivas (FE). Lezak (1982) las define como aquellas capacidades mentales necesarias para formular objetivos, planificar cómo alcanzarlos y llevar a cabo los planes de manera efectiva; son el centro de todo lo socialmente útil, permiten realizar actividades de mejora, constructivas y creativas. Así, las FE engloban diferentes funciones cognitivas que permiten la integración multimodal de información para construir una representación de la situación y seleccionar las respuestas más adecuadas según nuestros objetivos, permitiendo el control voluntario y flexible de la conducta (TirapuUstárroz, García-Molina, Luna-Lario, RoigRovira \& Pelegrín-Valero, 2008).

Al mismo tiempo, esta habilidad para inhibir 0 demorar respuestas preponderantes, que permite al individuo modificar su curso de acción en condiciones de conflictos, ya sea a nivel cognitivo, emocional o motor, se denomina Control Ejecutivo (CE), el cual puede ser evaluado por medio de dos dimensiones de las FE: el componente frío relacionado a procesos neuropsicológicos como la inhibición, la memoria de trabajo, el cambio de reglas y la planeación de una respuesta; mientras que 
el componente cálido implica la regulación y la motivación, obliga al individuo a reevaluar el significado motivacional de un estímulo, al tiempo que le permite responder rápidamente a los estímulos emocionales (Martínez \& Manoiloff, 2010; Zelazo \& Müller, 2002).

Figner et al. (2009) crearon la prueba Columbia Card Task (CCT) para medir el componente emocional (versión cálida) y el componente cognitivo (versión fría) en el proceso de toma de decisiones $y$ demostraron que las pruebas de funciones ejecutivas de orden superior que involucran habilidades tales como la planificación, resolución de problemas novedosos, inflexibilidad y perseverancia se correlacionan con el uso de información sólo en la versión fría. Markiewicz y Kubinska (2015) utilizaron esta prueba y demostraron que, en la versión cálida, la emoción interfiere con el procesamiento de la información porque perjudica la participación de procesos basados en reglas que requieren recursos de memoria de trabajo. De esta manera, el CCT muestra que la interacción emoción-cognición modula la toma de decisiones que puede ser guiada hacia objetivos adaptativos.

Recapitulando, cuando el estudiante ingresa a la universidad inicia un proceso de adaptación social, por ello es importante que desarrolle aspectos afectivos y cognitivos que fomenten su capacidad crítica, creadora y resolutiva para obtener un mejor desempeño académico y disminuir problemáticas escolares, como el abandono escolar. Al respecto, Tinto (1989) señala que, en las etapas iniciales de ingreso a la universidad es más frecuente la deserción escolar y, es en este momento donde las instituciones pueden implementar estrategias como sesiones de asesoría y orientación, grupos de estudio y tutorías académicas que permitan a los estudiantes superar los obstáculos en esta etapa de transición, tener un mejor desempeño académico y así evitar el abandono temprano.

El presente estudio tiene como propósito analizar en qué medida la competencia socioemocional y la toma de decisiones inciden en la deserción de los estudiantes universitarios.

\section{Método}

Este estudio tuvo un enfoque cuantitativo, con alcance exploratorio, transversal dentro de un diseño no experimental. Se midió la competencia socioemocional bajo tres subdominios: la resiliencia, la regulación emocional y la conducta prosocial; en la toma de decisiones se consideró el componente emocional y el componente cognitivo.

\section{Participantes}

La muestra se conformó de manera no aleatoria e intencional, con un total de 227 estudiantes de diferentes facultades de la Universidad Autónoma del Estado de Morelos. El promedio de edad de los estudiantes fue de 21.52 años con una desviación estándar de 2.48 años. El género y grado escolar de los participantes se indican en las tablas 1 y 2 . 
Tabla 1

Porcentaje de participantes por género

\begin{tabular}{ccc}
\hline Género & Participantes & $\%$ \\
\hline Mujeres & 156 & 68.7 \\
Hombres & 71 & 31.3 \\
\hline
\end{tabular}

Tabla 2

Porcentaje de estudiantes por semestre escolar

\begin{tabular}{ccc}
\hline Semestre & Participantes & $\%$ \\
\hline $2^{\circ}, 3^{\circ}{\mathrm{y} 4^{\circ}}^{\circ}, 8^{\circ} \mathrm{y}^{\circ}$ & 105 & 46.3 \\
& 122 & 57.7 \\
\hline
\end{tabular}

\section{Instrumentos}

Escala de medición de la resiliencia con mexicanos (RESI-M). Esta escala fue adaptada y validada por Palomar y Gómez (2010). Comprende 43 ítems que valoran la resiliencia en cinco factores: fortaleza $y$ confianza en sí mismo, competencia social, apoyo familiar, apoyo social y estructura. Las opciones de respuesta fueron (1totalmente en desacuerdo, 2-en desacuerdo, 3-de acuerdo y 4-totalmente de acuerdo). Estudios previos mostraron un alfa de Cronbach de $\alpha=.93$.

Inventario de competencias socioemocionales para adultos (ICSE). Se utilizaron las subescalas de regulación emocional y prosocialidad de Mikulic, Crespi \& Radusky (2015). Esta escala consta de 72 ítems, la escala de respuesta para fines de esta investigación se cambió de 5 a 4 opciones: 1-totalmente en desacuerdo, 2-en desacuerdo, 3-de acuerdo y 4-totalmente de acuerdo. El índice de confiabilidad obtenido fue de $\alpha=.68$.
Columbia Card Task (CCT). Es un instrumento que mide el comportamiento de los procesos de toma de decisiones por medio de dos tareas que evalúan el componente emocional y el componente cognitivo (Figner, 2009). Para el componente emocional se muestran las cartas con una cara sonriente que induce a ganar puntos y corresponde a las decisiones con componente emocional; mientras que el componente cognitivo se relaciona con las caras tristes que llevan a pérdidas considerables de puntos, correspondiente a las decisiones con mayor componente racional. A menor pérdida de puntos, mayor razonamiento al momento de elegir (Avilés, 2019).

Su versión original está disponible en idioma inglés con traducción únicamente al francés, italiano y alemán; por lo tanto, para adaptar la aplicación al idioma español, se utilizaron las siguientes bibliotecas de Python: 24.1. Tkinter- Python interface to Tcl/Tk- Python 2.7.18rc1 documentation, como kit de herramienta de los diversos comandos para la ejecución de la aplicación. Random - Generate pseudo- 
random numbers -Python 3.8.2 documentación, con este módulo se implementó el rango y la generación de números pseudoaleatorios para la distribución y secuencia de las cartas de pérdida (o incorrectas) del juego, de acuerdo al diseño factorial: a) probabilidad de pérdida (1 o 3 tarjetas de pérdida), b) cantidad de ganancia (10 o 30 puntos por tarjeta de ganancia) y c) cantidad de pérdida (250 o 750 puntos por tarjeta de pérdida). Por último, para escribir datos de cada intento y round (número de cartas volteadas, cantidad de pérdida, cantidad de ganancia); y exportar los datos en archivos de Excel (.xls) de cada uno de los participantes, se utilizó xlxt documentation -xlw 1.3.0

El software fue probado previamente con 20 estudiantes de licenciatura en el centro de cómputo de la Facultad de Biología de la UAEM.

Rendimiento académico. Se midió con el promedio acumulado de las calificaciones obtenidas durante su carrera, por lo tanto, es una puntuación intervalar (de 0 a 10 ).

\section{Procedimiento}

Los instrumentos fueron digitales, se aplicaron en un centro de cómputo, de manera grupal y contrabalanceada; de acuerdo a Vogt y Jonhson (2011) es una técnica de control agregada para adicionar validez a los resultados. Los participantes ingresaron a la aplicación mediante un número de usuario, protegiendo así su confidencialidad, y firmando un consentimiento informado para aceptar su participación. Los datos recabados se analizaron con base en estadísticos descriptivos y bivariados mediante el programa SPSS, versión 25. Con el submódulo de AMOS se realizó un análisis estructural, tomando en cuenta aspectos teóricos y empíricos. Se establecieron tres dimensiones para la competencia socioemocional: resiliencia, regulación emocional y conducta prosocial, los cuales son aspectos emocionales que interfieren con el procesamiento de la información durante la toma de decisiones, en la que interactúa un componente emocional y un componente cognitivo (Figura 1). Las rutas entre las variables se establecieron con base en los análisis de correlación bivariados obtenidos inicialmente y el comportamiento de los índices de ajuste del modelo. El método de estimación utilizado en el modelo estructural fue máxima verosimilitud.

\section{Resultados}

En los estadísticos descriptivos de las competencias socioemocionales se destacó que la media es mayor en la resiliencia, seguida de la conducta prosocial y la regulación emocional (tabla 3). En la toma de decisiones predominó el componente cognitivo con una media de 15.73 en comparación al componente emocional $(\mu=10.19)$. El rendimiento académico de la muestra es de 8.80 . 
Tabla 3

Variables de estudio

\begin{tabular}{ccccc}
\hline Dimensión & $\boldsymbol{\mu}$ & Mínimo & Máximo & DE \\
\hline Resiliencia & 3.58 & 2.49 & 4.00 & 0.28 \\
Regulación emocional & 2.14 & 1.50 & 3.38 & 0.28 \\
Conducta prosocial & 2.44 & 1.60 & 3.20 & 0.29 \\
Componente emocional & 10.19 & 4.38 & 16.29 & 2.16 \\
Componente cognitivo & 15.73 & 1.58 & 30.38 & 6.26 \\
Rendimiento académico & 8.80 & 6.00 & 10.00 & .60 \\
\hline
\end{tabular}

Posteriormente, se calcularon los coeficientes de correlación de Pearson entre las competencias socioemocionales y la toma de decisiones. Los resultados obtenidos se muestran en la Tabla 4, en la que se muestra una correlación media y negativa entre la resiliencia y la regulación emocional $(r=-.336,<.01)$; esto es, que a mayor resiliencia, menor regulación emocional; mientras que entre el componente emocional y el componente cognitivo existe correlación positiva $(r=$ $.431,<.01)$.

Tabla 4

Resultados obtenidos de las correlaciones de Pearson entre las variables de estudio

\begin{tabular}{ccccc}
\hline Dimensión & $\begin{array}{c}\text { Regulación } \\
\text { emocional }\end{array}$ & $\begin{array}{c}\text { Conducta } \\
\text { prosocial }\end{array}$ & $\begin{array}{c}\text { Componente } \\
\text { emocional }\end{array}$ & $\begin{array}{c}\text { Componente } \\
\text { cognitivo }\end{array}$ \\
\hline Resiliencia & $-.336^{* *}$ & $-.159^{*}$ & -0.086 & 0.036 \\
Regulación emocional & & $.140^{*}$ & 0.030 & -0.036 \\
Conducta prosocial & & & -0.116 & 0.049 \\
Componente emocional & & & & $.431^{* *}$ \\
\hline
\end{tabular}

*Correlación significativa al nivel .05 (bilateral)

**Correlación significativa al nivel .01 (bilateral)

Por último, se especificó un modelo estructural con las trayectorias expuestas en la figura 1, estimando los parámetros a través del método de máxima verosimilitud. De acuerdo con las relaciones que guardan las competencias socioemocionales, la toma de decisiones y el rendimiento académico, se evaluó el ajuste del modelo, el cual mostró índices de ajuste satisfactorios (Ruíz, Pardo y San Martín, 2010) presentados en la tabla 5 . 
Figura 1. Modelo estructural de las relaciones entre las competencias socioemocionales y la toma de decisiones en estudiantes universitarios.

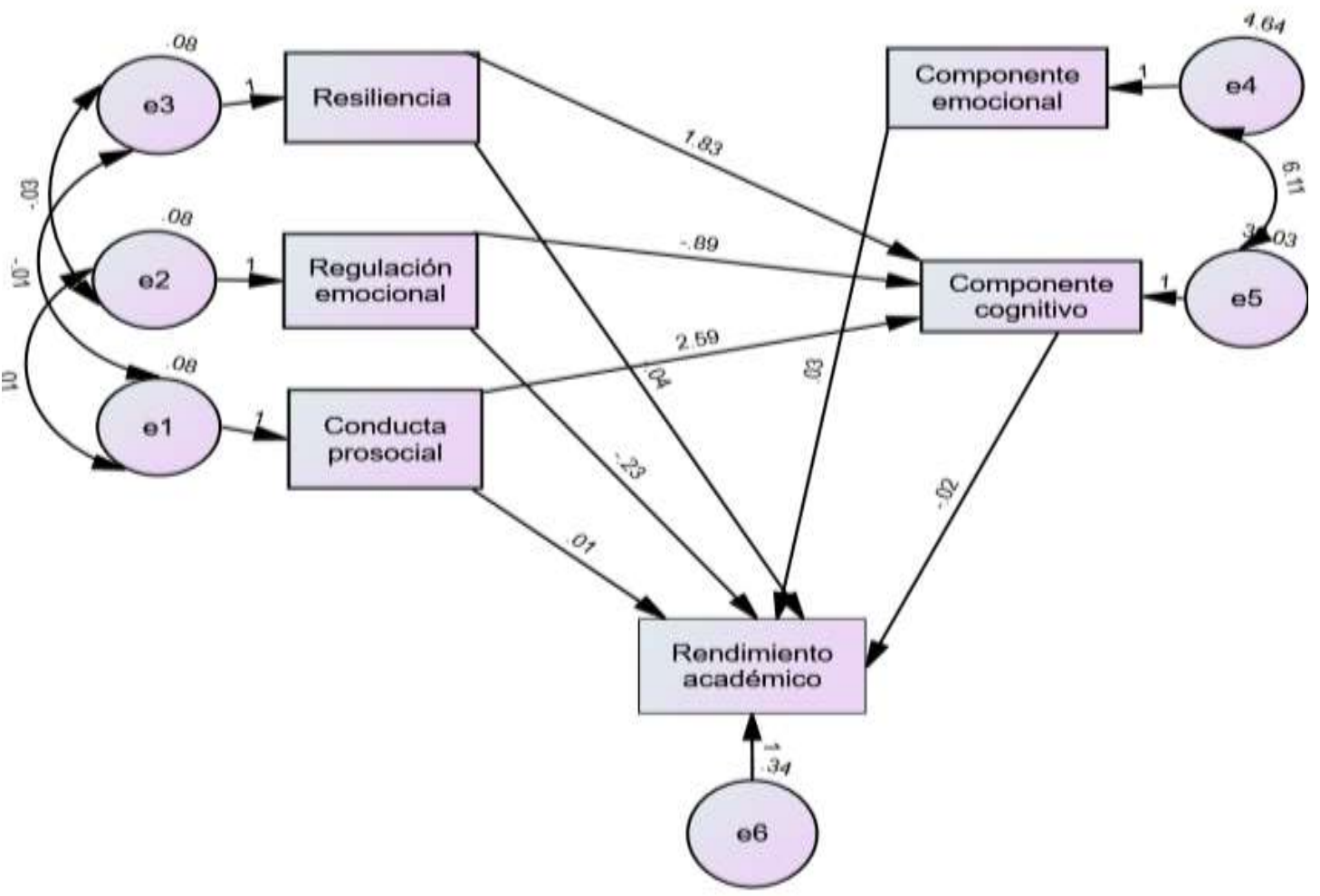

Fuente: Elaboración propia

Tabla 5

Índices de ajuste para el modelo de ecuaciones estructurales

\section{Esperado Obtenido}

\section{Ajuste absoluto}

Chi-cuadrado ( $\chi 2)$

$>0,05$

Razón Chi-cuadrado/Grados de libertad $(\chi 2 / g l)$

$<3$

\section{Ajuste comparativo}

Índice de bondad de ajuste comparativo (CFI)

Índice no normalizado de ajuste Tucker-Lewis (TLI)

Índice de ajuste normalizado (NFI)

$\begin{array}{ll}\geq 0,95 & 0.969 \\ \geq 0,95 & 0.844 \\ \geq 0.95 & 0.944\end{array}$

\section{Ajuste parsimonioso (NFI)}

Corregido por parsimonia (PNFI)

Próximo a 1

0.189

\section{Otros}

Índice de bondad de ajuste (GFI)

$\begin{array}{ll}\geq 0.95 & 0.992 \\ \geq 0.95 & 0.942\end{array}$

Índice de bondad de ajuste corregido (AGFI)

Índice residual de la raíz cuadrada media (RMR)

Próximo a 0

0.186

Error cuadrático media de aproximación (RMSEA) 


\section{Discusión}

Con base en los resultados obtenidos en este estudio, se puede afirmar que, entre los subdominios de la competencia socioemocional, destacó la resiliencia, esto significa que el estudiante utiliza sus recursos personales para adaptarse y sobreponerse a las condiciones adversas a las que está expuesto (Moskovitz, 1983). Un factor relevante de la resiliencia es la sociabilidad, al respecto, Vygotsky (1986) indicó la importancia de la interacción social para el aprendizaje y el desarrollo. De tal manera que, mediante un alto nivel de interacción e integración de los estudiantes en su entorno universitario, se favorece el compromiso de permanecer en la institución educativa, evitando así el abandono prematuro.

Por otra parte, en los resultados, predominó el componente cognitivo en la toma de decisiones de los estudiantes universitarios. De acuerdo a Brand et al. (2005) en el componente cognitivo se asume que la información relacionada con las características de las decisiones a tomar deviene de la memoria a largo plazo, esta información sería monitoreada por las FE, como la inhibición, el cambio de reglas y la planeación de una respuesta, que también son necesarias para categorizar las opciones ofrecidas en la situación, y en donde la memoria de trabajo tendría el rol de combinar la información recibida. En tal sentido, se interpreta que los estudiantes muestran una mayor capacidad de integración y análisis de información en la toma de decisiones; esto representa el potencial necesario que deben mantener e incrementar durante sus estudios universitarios para ser capaces de responder a situaciones complejas $\mathrm{y}$ diversas.

En cuanto a la relación entre la resiliencia, la regulación emocional, la conducta prosocial y el componente cognitivo de la toma de decisiones, se encontró una relación estadísticamente significativa. Sobre esto último, considerando la perspectiva de la cognición social, Maldonado (2019) señala que ésta comprende desde el procesamiento de emociones básicas hasta la compleja toma de decisiones, las cuales se ubican, en su mayoría, dentro de un determinado contexto social. Por lo tanto, se considera que un alto nivel de competencia socioemocional permite el intercambio de señales sociales que favorecen la obtención de información afectiva entre los estudiantes, y a partir de esa información tomar decisiones que se manifiesten en su conducta.

Sobre lo expuesto anteriormente, algunos autores (Butman \& Allegri, 2001; Adolphs, 2003; Damasio, 2006) consideran que las emociones, su identificación, expresión y manejo son un componente de la cognición social, además de que facilitan o dificultan la toma de decisiones frente a las interacciones sociales. Por ejemplo, Goleman (1996) señala que las emociones fuertes pueden ser una fuente poderosa para deshabilitar la capacidad de mantener una memoria de trabajo, la cual es un componente cognitivo de eficacia en el manejo del comportamiento en el aula. A su vez, en un estudio con estudiantes 
universitarios, Pérez (2019) identificó que los resultados obtenidos a nivel de función ejecutiva-dorsolateral y su relación con el rendimiento académico indican que, gracias a la memoria de trabajo, las personas pueden generar la consolidación de nuevos aprendizajes.

Lo anterior representa un área de oportunidad en la formación de los estudiantes universitarios, es decir, mediante el desarrollo de las habilidades socioemocionales se favorece la toma de decisiones para regular el comportamiento dentro del aula y mejorar así el rendimiento académico, de tal manera que se consolide el aprendizaje de los estudiantes y su permanencia en la universidad. Al respecto, Wright (2005) afirma que el comportamiento y el aprendizaje están vinculados: lograr un efectivo manejo del comportamiento en el aula tomando en cuenta nuestras propias emociones y las de los demás, dará como resultado mejores relaciones y mejores entornos de aprendizaje.

Una de las limitaciones del presente estudio fue su diseño transversal que no permitió observar la relación de las variables a través del tiempo, ya que de acuerdo con Chernyshenko, Kankaraš y Drasgow (2018) las competencias socioemocionales son altamente maleables en función de una variedad de factores individuales y contextuales, por lo tanto, los estados afectivos de los estudiantes pueden variar en función de los constantes cambios de adaptación social que surgen en su formación educativa, lo cual contribuye a experiencias de aprendizaje significativo que generan nuevas interpretaciones cognitivas de sus sensaciones y percepciones.

Por ejemplo, desde las diferencias individuales, podrían considerarse variables como la autorregulación, los rasgos de personalidad y la memoria de trabajo. Entre los factores contextuales que podrían determinar las habilidades sociales de los estudiantes dentro del ambiente escolar se encuentran los docentes, la relación con sus grupos de iguales y la normatividad de la institución. De igual forma, es recomendable que en estudios posteriores se incorporen variables socioeconómicas que pueden influir sobre el contexto estudiantil. Esto permitirá integrar factores de tipo familiar y social asociados con el rendimiento académico y la permanencia de los estudiantes, lo cual podría evitar la deserción universitaria.

\section{Conclusiones}

En conclusión, este estudio destaca que, en el ambiente universitario, mediante la cognición social y el desarrollo de habilidades socioemocionales en los estudiantes se promueve un compromiso de permanencia en la institución, esto impacta en su toma de decisiones durante el proceso de aprendizaje a lo largo de la vida.

Cuando el estudiante utiliza sus habilidades afectivas y cognitivas en etapas de adaptación, como lo es ingresar a la universidad, se sobrepone a situaciones desfavorables, así se confirma que, la relación entre el componente afectivo y el componente cognitivo permite un mejor manejo del comportamiento académico. 
Por ello, se considera que la competencia socioemocional incluye componentes tales como el autocontrol, la memoria de trabajo y la regulación emocional, los cuales forman parte de las Funciones Ejecutivas.

Sobre esto último, la comprensión de las correlaciones neuroanatómicas de las funciones cognitivas sirve para optimizar el

\section{Referencias}

Adeyemi, T. 0. (2008). Predicting student's performance in senior secondary certificate examinations from performance in junior secondary certificate examinations in Ondo State, Nigeria. Humanity \& Social Sciences Journal, 3, 26-36. Https://doi.org/10.4314/ajesms.v $\underline{4 i 1.38622}$

Adolphs, R. (2003). Cognitive neuroscience of human social behaviour. Nature Reviews, 4, pp. 165-178. Https://doi.org/10.1038/nrn1056

Almlund, M., Duckworth, A. Heckman, J. \& Kautz, T. (2011). Personality Psychology and Economics. NBER Working Paper, 16822, 1-252

Ayala, M. y Atencio, I. (2018). Retención en la educación universitaria en Chile. Aplicación de un modelo de ecuaciones estructurales. Revista de la Educación Superior, 47 (186), 93118.

https://doi.org/10.36857/resu.20 $\underline{18.186 .350}$

Bisquerra, R. (2003). Educación emocional y competencias básicas para la vida. rendimiento cognitivo o acelerar el proceso de aprendizaje mediante la generación de estrategias derivadas de esa comprensión. De ahí la importancia de diseñar intervenciones de entrenamiento cognitivo.

Revista de Investigación Educativa, $21 \quad$ (1), 7-43. Https://revistas.um.es/rie/article/ view/99071

Bisquerra, R. y Pérez, N. (2007). Las competencias emocionales. Educación XXI, 10, 61-82. Http://espacio.uned.es/fez/eserv/bibliune d:EducacionXXI-2007numero1023

Brand, M, Fujiwara, E., Borsutzky, S., Kalbe, E., Kessler, J., \& Markowitsch, H. J. (2005). Decision-Making Deficits of Korsakoff Patients in a Ne Gambling Task with Explict Rules: Assciations with Executive Functions. Neuropsychology, 19(3), 267-277. Https://doi.org/10.1037/08944105.19.3.267

Brody, G.H., Chen, Y.F., Murry, V.M., Ge, X., Simons, R., Gibbons, F.X., Gerrard, M. y Cutrona, C.E. (2006). Perceived Discrimination and the Adjustment of African American Youths: A FiveYear Longitudinal Analysis with Contextual Moderation Effects. Child development, 7(5), 1170- 
1189. Https://doi.org/10.1111/i.1 467-8624.2006.00927.x

Butman, J. \& Allegri, R.F. (2001). A cognição social e o córtex cerebral. Psicología: Reflexão e Crítica, 14 (2), pp. 275279.

Https://doi.org/10.1590/S010279722001000200003

Chernyshenko, OS., Kankaraš, M. y Drasgow, F. (2018). Social and emotional skills for student success and well-being: Conceptual framework for the OECD study on social and emotional skills. OECD Education Working, 173, 1-136. Https://doi.org/10.1787/db1d8e5 9-en

Damasio, A. (2006). El error de Descartes. La emoción, la razón y el cerebro humano. Barcelona, España: Crítica.

Dana L. H., Scott J. S. y Sunita, B. (2006). The company you keep: adolescent mobility and peer behavior. Sociological inquiry, 76(3), 397426.

https://doi.org/10.1111/j.1475682X.2006.00161.X

De Fruyt, F., B. Wille and O. John (2015), "Employability in the 21st Century: Complex (Interactive) Problem Solving and Other Essential Skills", Industrial and organizational Psychology, 8(2), 276281. https://doi.org/10.1017/iop. $\underline{2015.33}$

Eisenberg, N., \& Fabes, RA. (1998). Prosocial Development. En W. Damon (series ed.), N. Eisenberg
(Eds.). Handbook of Child Psychology: Social, Emotional, and Personality Development, (3, pp. 701-778). Nueva York: Wiley Https://doi.org/10.1002/9780470 147658.chpsy0311

Figner, B., Mackinlay, R., Friedrich, W. \& Weber, E.. (2009). Affective and Deliberative Processes in Risky Choise: Age Differences in Risk Taking in the Columbia Card Task. Journal of Experimental Psychology, 35 , 709-73. Https://doi.org/10.1037/a001498 $\underline{3}$

Garbanzo, V. (2007), Factores asociados al rendimiento académico en estudiantes universitarios, una reflexión desde la calidad de la educación superior pública. Revista educación, $31 \quad$ (1), 43-63. Https://doi.org/10.15517

Gaxiola, J. C., González, S. y Gaxiola, E. (2013). Autorregulación, Resiliencia y Metas Educativas: Variables Protectoras del Rendimiento Académico en Bachilleres. Revista Colombiana de Psicología, 22 (2), 241-252. Https://dialnet.unirioja.es/servlet/ articulo?codigo $=4703121$

Himmel, E. (2002). Modelos de análisis de la deserción estudiantil en la educación superior. Revista Calidad en la Educación: retención y movilidad estudiantil en la educación superior, 17 (2), 91-108. Http://dx.doi.org/10.31619/caled $\underline{\text { u.n17.409 }}$ 
Inglés, C. J. et al. (2009). Conducta prosocial y rendimiento académico en estudiantes españoles de Educación Secundaria Obligatoria. Anales de Psicología, 25(1), 93- 101. Https://www.redalyc.org/pdf/167 /16711594011.pdf

Isaza, L. y Henao, G.C. (2012). Actitudesestilo de enseñanza su relación con el rendimiento académico. International Journal of Psychological Research, 5 (1), 133141.

Https://www.redalyc.org/pdf/299 0/299023539015.pdf

Kautz, T., Heckman, J., Diris, R., Weel, B. \& Borghans, L. (2014). Fostering and Measuring Skills: Improving Cognitive and Non-Cognitive Skills to Promote Lifetime Success. OECD Education Working, 110, 1-86. Https://doi.org/10.3386/w20749

Knight, C. (2007). A resilience framework: perspectives for educators. Health Educ, 107, 543-555. Https://doi.org/10.3402/ejpt.v5.2 $\underline{5338}$

Lezak, M. (1982). The problema of assessing executive functions. Intternational Journal of Psychology, 17 , 281-297.

Https://doi.org/10.1080/0020759 8208247445

Luo J. \& Yu R. (2015). Follow the heart or the head? The interactive influence model of emotion and cognition. Front. Psychol, 6, 1-14.
Https://doi.org/10.3389/fpsyg.20 15.00573

Maldonado, G. (2014). ¿Qué determinan los diferentes tipos de emociones que registrar los seres humanos? En L.A. Rodríguez y E. J. Huaire (Ed.), Repensando la Psicología Educativa en la sociedad del conocimiento (pp. 87-95). Lima, Perú: Punto \& Grafía S.A.C

Maldonado, G. (2019). Diabetes Mellitus Tipo 2 y sus efectos sobre procesos de Cognición Social [Tesis de doctorado, Universidad Autónoma del Estado de Morelos]. Repositorio Institucional de Acceso Abierto de la Universidad Autónoma del Estado de Morelos.

Markiewicz, L. y Kubinska, E. (2015). Information Use Differences in Hot and Cold Risk Processing: When Does Information About Probability Count in the Columbia Card Task?. Frontiers in Psychology, 6, 17-27. Https://doi.org/10.3389/fpsyg.20 $\underline{15.01727}$

Martínez, MV., y Manoiloff, LMV. (2010). Evaluación Neuropsicológica de la Función Ejecutiva en Adolescentes con Diferentes Patrones de Consumo de Alcohol. Revista Argentina de Ciencias del Comportamiento, 2(1), 14-23. Https://revistas.psi.unc.edu.ar/ind ex.php/racc/article/view/5266/54 29

Mestre, J., \& Guil, R. (2012). La regulación de las emociones. Una vía a la 
adaptación personal y social. Madrid: Pirámide.

Mikulic, I., Crespi, M. y Radusky, P.. (2015). Construcción y validación del inventario de competencias socioemocionales para adultos (ICSE). Interdisciplinaria, 32, 307329.

Https://www.redalyc.org/pdf/180 /18043528007.pdf

Moskovitz, S. (1983). Love despite hate. New York: Schocken Books.

Palomar, L. y Gómez, V. (2010). Desarrollo de una escala de medición de la resiliencia con mexicanos (RESI-M). Interdisciplinaria, $\quad 27, \quad 7-22$. Https://www.redalyc.org/pdf/180 /18014748002.pdf

Pérez, K. (2019). Influence of the executive function in the academic performance of university students. The case of Fundación Universitaria Tecnológico Comfenalco. Revista Espacio, 40(8), 7-13. Http://www.revistaespacios.com/ a19v40n08/19400807.html

Pizarro, R. (1985). Rasgos y actitudes del profesor efectivo [Tesis de maestría, Pontificia Universidad Católica de Chile]. Repositorio Institucional de la Pontifica Universidad Católica de Chile.

Reimann, M. \& Bechara, A. (2010). The somatic marker framework as a neurological theory of decisiónmaking: Review, conceptual comparisons, and future neuroeconomics research. Journal of Economic Psychology, 31 (5), 767 776.

https://doi.org/10.1016/j.joep.201 $\underline{0.03 .002}$

Ruiz, M. A., Pardo, A. y San Martín, R. (2010). Modelos de ecuaciones estructurales. Papeles del Psicólogo, 31

34-45. Http://www.papelesdelpsicologo.e s/pdf/1794.pdf

Secretaría de Educación Pública (2020). Programa Construye-T, web: https://www.construyet.org.mx/ConstruyeT

Tinto, V. (1975). Dropout from Higher Education: A Theoretical Synthesis of Recent Research. Review of Educational Research, 45 (1), 89125.

Https://doi.org/10.3102/0034654 $\underline{3045001089}$

Tinto (1989) Tinto, V. (1988). Stages of Student Departure: Reflections on the Longitudinal Character of Student Leaving. The Journal of Higher Education, 59 (4), 438-455. Https://doi.org/10.1080/0022154 $\underline{6.1988 .11780199}$

Tirapu-Ustárroz, J., García-Molina, A., LunaLario, P., Roig-Rovira, T., \& PelegrínValero, C. (2008). Modelos de funciones y control ejecutivo (II). Rev neurol, 46(12), 742-750. Https://doi.org/10.33588/rn.4612 .2008252

Velasco, V., Suárez, G., Córdova, S., Luna, L. \& Mireles, S. (2015). Niveles de resiliencia en una población de 
estudiantes de licenciatura y su asociación con variables familiares y académicas. Revista Iberoamericana de Producción Académica y Gestión Educativa, (2), 1-23.

Http://doi.org/10.17081/psico.19. 36.1294

Vygotsky, L. S. (1986). Thought and language. Cambridge, MA: The Massachusetts Institute of Technology.

Vogt, W. P. \& Johnson, R. (2011). Dictionary of statistis \& methodology: A nontechnical guide for the social sciences. Thousand Oaks, CA: Sage.

Wright, D. (2005). There is no need to shout! The secondary teacher's guide to behaviour management. Cheltenham: Nelson Thornes Ltd.
Zelazo, PD., \& Müller, U. (2002). Executive function in typical development. In U. Goswami (Ed), Blackwell handbooks of developmental psychology. Backwell handbook of childhood cognitive development (pp. 445469). 